\title{
La sinistra italiana e gli ebrei: riflessioni su un dibattito storiografico
}

\author{
Alessandra Tarquini*
}

\begin{abstract}
The Italian Left and the Jews: reflections on a historiographical debate

The author inquires into the relationship between the Italian Left and the Jews, in light of the main contributions on the issue. Unlike the US and the rest of Europe, the topic has been studied in Italy only since the 1990s. In fact, from Marx until recent times, several left-wing intellectuals and politicians denied specificity to the problem in the name of proletarian internationalism and the labor movement tradition, believing it would be solved within the broader project to change society, the latter concerning the oppressed worldwide regardless of their cultural, religious, linguistic and national identity. After having analyzed the historiographical debate from the post-war era to present times, the author argues that the most conclusive way to study the issue is the method employed by George Mosse in 1971: i.e. to reconstruct the political and cultural relationship that the left-wing political family, in its different expressions, entertained with the Jews, in order to acquire knowledge of both their histories and focus on one of the most significant issues of the 20th century, namely the relationship between the most relevant revolutionary political project of contemporary age and the most ancient minority of the world.
\end{abstract}

Keywords

Left, Jews, Anti-Semitism, Italian Intellectuals, Cultural history, historiography

Ricevuto 9 aprile 2019 - Accettato 4 giugno 2019

\section{Introduzione}

Come hanno mostrato autorevoli studiosi, ormai da diversi anni, dopo la seconda guerra mondiale, la storia degli ebrei è stata relegata ai margini della storiografia, dove è rimasta fino a quando la cultura occidentale è riuscita a elaborare la memoria delle persecuzioni ${ }^{1}$. In questo orizzonte,

* Sapienza Università di Roma. alessandra.tarquini@uniroma1.it

${ }^{1}$ Cfr. A. Rossi Doria, Memoria e storia. Il caso della deportazione, Rubbettino, Soveria Mannelli, 1998; M. Toscano, Ebraismo e antisemitismo in Italia, FrancoAngeli, Milano, 2003; G. Schwarz, “A proposito di una vivace stagione storiografica: letture dell'emancipazione

Mondo contemporaneo, n. 2-2019, ISSN 1825-8905, ISSNe 1972-4853 
all'indomani del conflitto, i rapporti fra la sinistra e gli ebrei non costituirono un tema di ricerca perché nessuno fra gli studiosi e i protagonisti - né gli ebrei, né gli esponenti del variegato mondo della sinistra - era disponibile a mettere in discussione una convinzione diffusa e, in buona parte corrispondente al vero, e cioè che la storia degli uni e quella degli altri avevano molto in comune: l'emancipazione degli ebrei era iniziata con la Rivoluzione francese e con l'affermarsi dei principi illuministi in Europa e nel mondo; il sionismo moderno, che portò alla nascita di Israele, affondava le sue origini nella cultura politica della Seconda Internazionale; all'inizio del XX secolo il socialismo dell'Est europeo aveva autorevoli esponenti e dirigenti fra gli intellettuali ebrei; ma soprattutto, dagli anni Trenta, gli ebrei e la sinistra avevano condiviso la lotta contro un nemico terribile combattendo contro il nazifascismo e contro le persecuzioni culminate con la Shoah. Dunque, la loro relazione non costituiva un problema e, tantomeno, una questione storiografica.

In realtà, questo silenzio non è stato uniforme nel tempo e nei diversi contesti nazionali: mentre in America e in alcuni paesi europei, dalla fine degli anni Sessanta, il tema ha riscosso l'interesse degli storici, dei sociologi e degli studiosi del pensiero politico, in Italia è diventato parte del dibattito storiografico solo dagli anni Novanta e con alcune peculiarità. Questo saggio è diviso in tre parti: la prima è dedicata alle domande della politica e cioè ai saggi pubblicati da giornalisti e uomini politici, dagli anni Settanta a giorni recenti; la seconda ai lavori degli storici sulla sinistra e il conflitto arabo-israeliano; e la terza ai contributi di quanti hanno studiato il pensiero politico degli esponenti della tradizione marxista e indagato le rappresentazioni dell'antisemitismo proposte da socialisti e comunisti nel corso del Novecento.

\section{Le domande della politica}

In America e in Europa, la riflessione sui rapporti fra la sinistra e gli ebrei è iniziata negli anni Cinquanta con gli studi pionieristici di Edmund

ebraica negli ultimi vent'anni", Memoria e ricerca, 19, 2005, pp. 159-174; Id., "The Reconstruction of Jewish life in Italy after World War II", Journal of Modern Jewish Studies, 8, 2009, pp. 360-377; I. Pavan, "Gli storici e la Shoah in Italia", in M. Flores, S. Levis Sullam, M.-A. Matard-Bonucci, E. Traverso (a cura di), Storia della Shoah in Italia. Vicende, memorie, rappresentazioni, Utet, Torino, 2010, II, pp. 135-164; R. Gordon, Scolpitelo nei cuori. L'Olocausto nella cultura italiana (1944-2010), Bollati Boringhieri, Torino 2013; C. Ferrara degli Uberti, "Gli ebrei nell'Italia contemporanea", Nuova informazione bibliografica, 4, 2013, pp. 827-840. 
Silberner sul socialismo europeo dell'Ottocento ${ }^{2}$. È proseguita, all'inizio degli anni Settanta, con i primi lavori sui diversi contesti nazionali, come quelli di George L. Mosse sulla questione ebraica nella socialdemocrazia tedesca, o quelli di Annie Kriegel per il caso francese ${ }^{3}$. È andata avanti nei decenni successivi con i contributi sul pensiero politico di matrice socialista di diversi autori fra cui Robert Wistrich, Zeev Sternhell, Pierre Birnbaum e Enzo Traverso ${ }^{4}$. E, nei tempi recenti, ha continuato a suscitare l'attenzione di storici, filosofi e sociologi della politica. Di fatto, in Europa e in America, chi ha studiato la relazione fra la sinistra e gli ebrei si è confrontato con tre temi: 1) le riflessioni dei principali esponenti della cultura politica di sinistra, dal socialista utopista Charles Fourier alla Scuola di Francoforte; 2) la reazione della sinistra di fronte all'antisemitismo, sia quello degli anni Trenta, sia quello presente nello scenario internazionale della Guerra Fredda; 3) il rapporto dei partiti della tradizione socialista e comunista con il sionismo, con particolare riguardo a Israele e al conflitto con i paesi arabi per il periodo che segue il 1948.

${ }^{2}$ E. Silberner, "Charles Fourier on the Jewish Question", Jewish Social Studies, 4, 1946, pp. 245-266; Id., "The Attitude of Fourierist School towards the Jews", ibid., 4, 1947, pp. 339-362; Id., "Proudhon's Judeophobia", Historia Judaica, X, 1948, pp. 61-80; Id., "Friedrich Engels and the Jews", Jewish Social Studies, 4, 1949, pp. 323-342; Id., "Was Marx an Anti-Semitic?", in E. Mendelsohn (ed.), Essential Papers on Jews and the Left, New York University Press, New York, 1997, pp. 361-401 (reprinted from Historia Judaica, XI, 1949); E. Silberner, "British socialism and the Jews", ibid., XIV, 1952, pp. 27-52; Id., "French socialism and the Jews", Historia Judaica, XV-XVI, 1953-1954, pp. 4-38; Id., "Anti-Jewish Trends in French Revolutionary Sindacalism", Jewish Social Studies, XV, 3-4, 1953, pp. 195-202; Id., "Anti-Semitism and Philo-Semitism in the Socialist International", Judaism, 2 , 1953, pp. 117-122; Id., "German Social Democracy and the Jewish Problem Prior to World War I", Historia Judaica, 1953, pp. 3-48; Id., Western European Socialism and the Jewish problem 1800-1918. A selective bibliography, Hebrew University, Jerusalem, 1955.

${ }^{3}$ G.L. Mosse, Germans and Jews: The Right, the Left, and the Search for a "Third Force" in Pre-Nazi Germany, Howar Fertig, New York, 1970, pp. 144-170; Id., "German Socialists and the Jewish Question in the Weimar Republic", The Leo Baeck Institute Year Book, 1, 1971, pp. 123-151; Id., "Left Wing Intellectuals and the Jewish Problem in the Thirties and in the Sixties", Dispersion and Unity, 17-18, 1973, pp. 106-116; Id., Communismes au miroir français: temps, cultures et sociétés en France devant le communisme, Gallimard, Paris, 1974; A. Kriegel, "Le socialisme au miroir juif", in Réflexion sur les questions juives, Hachette, Paris, 1984.

${ }^{4}$ R.S. Wistricht (ed.), The Left against Zion. Communism, Israel and the Middle East, Vallentine Mitchell, London-New York, 1979; W. Cohen, I. Wall, "French Communism and the Jews", in F. Malino, B. Wasserstein (eds.), The Jews in Modern France, Brandeis University Press, Hanover, 1985, pp. 81-102; Z. Sternhell, "Roots of Popular Anti-Semitism in the Third Republic", ibid., pp. 103-134; P. Birnbaum, "Nation, Etat et culture: l'exemple du sionisme", Communication, 45, 1987, pp. 157-170; E. Traverso, Les marxistes et la questione juive: histoire d'un débat (1843-1943), La Breche Pec, Montreuil, 1990. 
In Italia la riflessione è nata e si è sviluppata diversamente, non mostrando la stessa ricchezza di temi. Dall'inizio degli anni Settanta, infatti, il tema è stato oggetto di sintesi giornalistiche, o di analisi circoscritte a brevi periodi, concentrate sulle reazioni degli esponenti della sinistra rispetto alle varie fasi del conflitto arabo-israeliano 5 . In particolare, dopo la guerra dei Sei giorni, del giugno 1967, gli stessi partiti e movimenti sono stati i protagonisti di ricerche dedicate al conflitto mediorientale e soprattutto alla causa palestinese. Del rapporto fra la sinistra e l'antisemitismo, della relazione fra il socialismo e la questione ebraica, di come tutto questo avesse influito nella costruzione dei rapporti fra gli esponenti della tradizione socialista e comunista e Israele, nel dibattito italiano degli anni Settanta non vi fu traccia, se non in alcune eccezioni che, tuttavia, non mutarono il panorama degli studi.

Nel 1970 un esponente del Partito comunista italiano, Luciano Ascoli, pubblicò un piccolo pamphlet in cui affrontò temi che in Europa e in America avevano già trovato accoglienza: l'identità dello Stato di Israele, i rapporti fra l'antisionismo e l'antisemitismo e la riflessione sul testo di Marx Sulla questione ebraica del $1844^{6}$. In quelle pagine, Ascoli ricordò che la

${ }^{5}$ Cfr. L. Ascoli, Sinistra e questione ebraica. Antisionismo fase suprema dell'antisemitismo, La Nuova Italia, Firenze, 1970; M. Massara (a cura di), Gli studi sul pensiero di Marx. Il marxismo e la questione ebraica, Edizioni del Calendario, Milano, 1972; A. Di Nola, Antisemitismo in Italia 1962/1972, Vallecchi, Firenze, 1973; M. Achilli, I socialisti tra Israele e Palestina. Dal 1892 ai nostri giorni, Marzorati, Settimo milanese, 1989; M. Molinari, La sinistra e gli ebrei in Italia. 1967-1993, Corbaccio, Milano, 1995. Sul Pci cfr. L. Riccardi, Il "problema Israele». Diplomazia italiana e Pci di fronte allo Stato ebraico (1948-1973), Guerini, Milano, 2006; Id., L'internazionalismo difficile. La "diplomazia” del PCI e il Medio Oriente dalla crisi petrolifera alla caduta del muro di Berlino (1973-1989), Rubbettino, Soveria Mannelli, 2013; G. Santese, "Il Partito comunista italiano e la questione palestinese (1945-1956): L'Unità e Rinascita, Mondo contemporaneo, 2, 2007, pp. 63-104. Sul Psi cfr. G. Tortorelli, "L'Affaire Dreyfus e i socialisti italiani", Mélanges de l'Ecole française de Rome, 1, 1989, pp. 498-513; F. Biagini, "Sionismo, marxismo e socialismo italiano (1897-1917)", Annali del Dipartimento di Scienze storiche geografiche e sociali, VIII, 1991-1992, pp. 311-345; A. Cavaglion, "Il sionismo nella stampa socialista di fine Ottocento. Osservazioni preliminari”, in Italia Judaica. Gli ebrei nell'Italia unita 1870-1945. Atti del IV convegno internazionale, Ministero per i beni culturali e ambientali, Roma, 1993, pp. 223-236; M. Toscano (a cura di), Ebraismo, sionismo e antisemitismo nella stampa socialista italiana. Dalla fine dell'Ottocento agli anni Sessanta, Marsilio, Venezia, 2004. Sulla sinistra: M. Di Figlia, Israele e la sinistra. Gli ebrei nel dibattito pubblico italiano dal 1945 a oggi, Donzelli, Roma, 2012; C. Brillanti, "La stampa della sinistra italiana e il conflitto arabo-israelo-palestinese. Dalla guerra dei Sei Giorni alla guerra dello Yom Kippur", Mondo contemporaneo, 2, 2013, pp. 9-52; Id., Le sinistre italiane e il conflitto arabo-israelopalestinese 1948-1973, Sapienza Università Editrice, Roma, 2018; V. Baldacci, 1967. Comunisti e socialisti davanti alla guerra dei sei giorni. La costruzione dell'immagine dello Stato di Israele nella sinistra italiana, Aska, Firenze, 2014; M. Nani, "Lavoratori antisemiti? Un sondaggio sul caso italiano (1860-1914)", Passato e presente, 101, 2017, pp. 101-122.

${ }^{6}$ L. Ascoli, Sinistra e questione ebraica, cit., p. 23.

"e" in
tondo e
virgolette
finale


nascita dello Stato d'Israele non era riconducibile all'antisemitismo degli anni Trenta. Nato a fine Ottocento dai nuclei di coloni che avevano raggiunto la Palestina, il sionismo non derivava soltanto da una reazione alle persecuzioni che avevano segnato la vita degli ebrei dalla fine del XIX fino al 1948. Si trattava di un movimento politico prodotto da una spinta nazionale che aveva permesso agli ebrei di diventare uno Stato e di interrompere un'antica tradizione. Secondo Ascoli, fra l'altro, l'idea che Israele esistesse per rispondere all'odio antiebraico degli anni Trenta e alla Shoah, lasciava intendere che quando fosse venuta meno l'ondata di antisemitismo, anche il nuovo Stato non avrebbe avuto motivo di esistere e ogni israeliano sarebbe potuto «ridiventare un normale ebreo», nel momento in cui si fosse smesso di perseguitarlo ${ }^{7}$. Per queste ragioni Ascoli criticò duramente quanto sostenuto pochi anni prima dallo storico marxista francese Maxime Rodinson. Nel 1967 in Israël fait colonial?, un articolo destinato ad avere molta fortuna, Rodinson scrisse che

la formation de l'Etat d'Israël sur la terre palestinienne est l'aboutissement d'un processus qui s'insère parfaitement dans le grand mouvement d'expansion européo-américain des XIXe et XXe siècles pour peupler ou dominer économiquement et politiquement les autres terres ${ }^{8}$.

L'anno successivo pubblicò Israël et le refus arabe, che venne tradotto in diverse lingue contribuendo a stimolare l'impegno politico di un'intera generazione in favore della causa palestinese. Nel testo Rodinson tornò ad accusare gli israeliani di aver dato vita ad un movimento colonialista, che aveva come basi «etnico-religiose» il «settarismo» e che, volta per volta, aveva cercato il sostegno delle potenze internazionali per perseguire una politica di guerra nel cuore del Medio Oriente ${ }^{9}$. Contro questa interpretazione, Ascoli obiettò che il sionismo non era una forma di colonialismo perché colonizzare un paese significava sfruttarne le risorse, drenando profitti verso la madrepatria.

Il libro scatenò un dibattito vivace all'interno del Pci e venne recensito duramente da Luca Pavolini, il direttore di Rinascita, che nell'ottobre 1973 tornò sull'argomento commentando quanto sostenuto dall'antropologo Al-

\footnotetext{
${ }^{7}$ Ibid., p. 41; L. Riccardi, Il «problema Israele», cit., pp. 306-308.

${ }^{8}$ M. Rodinson, "Israël fait colonial?", Temps Modernes, 253 bis, 1967, pp. 17-88; Id., Israël et le refus arabe. 75 ans d'histoire, Éditions du Seuil, Paris, 1968 (trad. it., Israele e il rifiuto arabo, Einaudi, Torino, 1969).

${ }^{9}$ Per le polemiche suscitate dall'intervento di Rodinson, cfr. M. Uzan, "Israël et les intellectuel français de 1967 à 1982", Controverses, 7, 2008, pp. 190-212.
}

togliere la virgola dopo it. 
fonso Di Nola in un lavoro sull'antisemitismo ${ }^{10}$. Nel suo studio Di Nola aveva indicato alcuni episodi di intolleranza comparsi nella sinistra italiana fra la fine degli anni Sessanta e il decennio successivo sostenendo che il confine fra l'antisemitismo e l'antisionismo si mostrava decisamente labile. A questo proposito, Pavolini scrisse:

La posizione duramente critica verso la linea espansionistica e annessionistica dei governanti dello Stato di Israele non è una scelta ideologica ma politica. Di Nola non è in grado di indicare un solo documento, un solo articolo, una sola parola proveniente dal movimento operaio, democratico, di sinistra italiano nel quale affiori una qualsiasi confusione tra antisionismo e antisemitismo, tra il termine ebreo e il termine israeliano ${ }^{11}$.

Come si può notare, si trattava di posizioni interne allo stesso mondo della sinistra, ma inconciliabili. Da questo punto di vista, l'antologia curata da Massimo Massara nel 1972, Il marxismo e la questione ebraica, fu decisamente più rappresentativa della cultura politica comunista dell'inizio degli anni Settanta, di quanto fosse il lavoro di Ascoli che pure era un esponente del $\mathrm{Pci}^{12}$. Pubblicato dalle edizioni del Calendario, un editore vicino al partito, il volume raccoglieva testi del pensiero marxista per mostrare la condanna del sionismo e l'impossibilità dei comunisti di essere antisemiti. Nell'introduzione Massara definì il sionismo un'ideologia imperialista che predicava l'unione di tutti gli ebrei in quanto tali, indipendentemente dalla loro collocazione di classe, e notò che nessuno come i comunisti avrebbe potuto combattere l'antisemitismo, dato che «il socialismo scientifico, il marxismo, è la concezione del mondo e della vita di coloro che si battono per la liberazione dell'uomo» ${ }^{13}$. Coerentemente con queste premesse, presentate come se fossero sufficienti a mostrare l'assenza di pratiche antisemite nella cultura e nella politica di sinistra, Massara sostenne che i sionisti cercavano di far accettare al movimento operaio un'ideologia borghese, reazionaria e nazionalista e di tenere in vita l'antisemitismo. Un'accusa certamente rozza, ma, evidentemente, non percepita come tale se Massara scrisse che «Hitler [aveva] avuto la funzione "provvidenziale" di salvare il popolo ebraico in quanto, con le sue persecuzioni, [aveva] contribuito a far rinascere e a tener desto in moltissimi ebrei un sentimento di identità e di

${ }^{10}$ Per le polemiche nel Pci, cfr. Archivio Partito Comunista Italiano, mf. 552 1887-1890. A.M. Di Nola, Antisemitismo in Italia 1962/1972, cit.; L. Riccardi, Il «problema Israele», cit., pp. 306-308.

${ }^{11}$ L. Pavolini, "Da che parte viene l'antisemitismo", L'Unità, 20 ottobre 1973.

${ }^{12}$ M. Massara (a cura di), Gli studi sul pensiero di Marx. Il marxismo e la questione ebraica, cit.

${ }^{13}$ Ibid., p. 20. 
comunità» ${ }^{14}$. È probabile che il variegato e complesso mondo della sinistra non avrebbe fatto sue queste affermazioni, anche se si trattava di una riflessione compatibile con la posizione che i comunisti avevano assunto rispetto al conflitto mediorientale e con quanto sostenevano dall'inizio degli anni Cinquanta.

In effetti, già da allora i rapporti fra la sinistra italiana e Israele avevano incontrato una serie di difficoltà, a differenza di quanto è stato sostenuto in un volume recente, che fa sua una riflessione ampiamente diffusa: «Sostenere il popolo ebraico nella creazione di un proprio Stato fu, infatti, considerato a lungo dall'opinione pubblica italiana, soprattutto quella parte maggiormente impegnata nel difendere la Resistenza e i suoi valori, una sorta di dovere morale, che derivava direttamente dalla lotta contro il nazifascismo» ${ }^{15}$. Se è vero che tutta la sinistra italiana, allo scoppiare della prima guerra arabo-israeliana, era stata compatta nel sostenere Israele, già alla fine degli anni Quaranta, con il venire meno dell'appoggio dell'Urss al nuovo Stato, cambiò posizione ${ }^{16}$. Durante la guerra di Suez del 1956, ad esempio, socialisti e comunisti avevano maturato un'avversione nei confronti della politica di Israele, sostenuto a quel punto dal solo partito socialdemocratico e dai repubblicani. Certamente la guerra dei Sei giorni esasperò i termini della questione. Come hanno rilevato diversi storici, dal 1967 cambiò una percezione dell'opinione pubblica internazionale: per la prima volta, da piccolo paese in guerra per difendere la propria esistenza, agli occhi di molti Israele si trasformò in uno Stato aggressore nel cuore del Mediterraneo. È noto, infatti, che alla fine della guerra del giugno 1967, gli israeliani occupavano tutto il Sinai, la striscia di Gaza, le alture del Golan e la Cisgiordania con Gerusalemme est. Avevano sconfitto le forze armate dell'Egitto, della Giordania e della Siria e in sei giorni avevano modificato l'equilibrio geopolitico della regione, rimanendo in attesa di scambiare terra in cambio di pace. E, soprattutto, avevano conquistato un'area tre volte e mezzo più grande di Israele stesso, abitata da oltre un milione di palestine$\mathrm{si}^{17}$. Nel 1967, a sostenere le ragioni dello Stato ebraico, nella sinistra ita-

${ }^{14}$ Ibid., p. 17.

${ }^{15}$ M. Simoni, A. Marzano, "Sessanta anni di relazioni politiche e culturali tra Italia e Israele", introduzione a Id. (a cura di), "Roma e Gerusalemme". Israele nella vita politica e culturale italiana (1949-2009), Ecig, Genova, 2010, p. 20.

${ }^{16}$ Cfr. M. Toscano, "1948. La fine di un pellegrinaggio bimillenario", in M. Toscano (a cura di), L'Italia racconta Israele 1948-2018, Viella, Roma, 2018, pp. 15-40; C. Brillanti, “1958. Le suggestioni del neoatlantismo e il fascino del kibbutz", ibid., pp. 41-66; A. Tarquini, "1968. Un anno dalla guerra dei Sei giorni, venti dalla nascita di Israele”, ibid., pp. 67-88.

${ }^{17}$ D. Caviglia, M. Cricco, La diplomazia italiana e gli equilibri mediterranei. La politica mediorientale dell'Italia dalla guerra dei Sei Giorni al conflitto dello Yom Kippur (19671970), Rubbettino, Soveria Mannelli, 2006, p. 24. 
liana vi erano: il partito socialdemocratico, il partito repubblicano, dal 1948 suoi tenaci sostenitori, e il partito socialista di Nenni che dall'inizio degli anni Sessanta aveva mutato il proprio atteggiamento abbandonando l'antisionismo degli anni Cinquanta. Il partito comunista era, invece, su una posizione di dura critica che non avrebbe abbandonato per molto tempo ${ }^{18}$.

In ogni caso, al di là delle scelte dei partiti di sinistra, che non sono oggetto di questo saggio, il dibattito restò legato alle posizioni politiche in campo, anche quando gli scenari internazionali e quelli interni cambiarono nettamente. Alla metà degli anni Ottanta, infatti, il Psi guidato da Bettino Craxi aveva assunto una posizione di chiaro sostegno alla causa palestinese, mitigando notevolmente la vicinanza a Israele espressa dalla segreteria di Pietro Nenni, mentre il Pci di Achille Occhetto aveva iniziato a smussare il proprio tradizionale filo-arabismo.

Anche allora la riflessione sui rapporti fra la sinistra e gli ebrei ricevette un forte stimolo dal mondo della politica e del giornalismo, come dimostra la prima ricostruzione complessiva dei rapporti fra un partito di sinistra italiano e Israele, pubblicata da Michele Achilli, un esponente della sinistra del partito socialista, amico personale di Yasser Arafat ${ }^{19}$. Basato sulla stampa di partito, il volume di Achilli mostrò le diverse politiche dei segretari del Psi, dando uno spazio particolare al secondo dopoguerra. Alla scelta filo-israeliana di Pietro Nenni, contrappose gli anni successivi nei quali lentamente il partito socialista si era spostato su una posizione filo-araba che aveva avuto in Craxi il suo maggiore esponente. Convinto che l'Italia dovesse assumere un nuovo ruolo nel Mediterraneo, e quindi rafforzare i tradizionali rapporti di amicizia con i paesi arabi, ma anche sensibile alla causa palestinese, che dagli anni Settanta era ormai al centro delle cronache internazionali, Craxi legò la sua presidenza del Consiglio all'impegno in favore dell' $\mathrm{Olp}^{20}$. In effetti, Achilli esprimeva una posizione molto vicina a quella del segretario del suo partito e molto distante da quanto sostenuto da Giorgio Gangi, un esponente del Psi milanese, sensibile alla causa israeliana, che pubblicò un piccolo volume in cui raccolse i suoi articoli sul rinascere dell'antisemitismo in Europa e in Italia, sul problema dei diritti umani degli ebrei sovietici e sulle incomprensioni della sinistra di fronte alla questione ebraica ${ }^{21}$.

Anche Maurizio Molinari intervenne nella discussione con un volume di taglio giornalistico che poneva l'accento sulle responsabilità dei diversi

\footnotetext{
${ }^{18}$ Per le posizioni della politica italiana nel 1968 cfr. A. Tarquini, “1968. Un anno dalla guerra dei Sei giorni, venti dalla nascita di Israele", cit.

${ }^{19} \mathrm{M}$. Achilli, I socialisti tra Israele e Palestina, cit.

${ }^{20}$ Ibid., p. 333.

${ }^{21}$ G. Gangi, Cinque anni di battaglie per Israele, Sugarco, Milano, 1987.
} 
esponenti della sinistra di fronte alla questione ebraica. Scegliendo come periodo di analisi gli anni 1967-1993, Molinari sostenne che in Italia, finito il secondo conflitto mondiale, il dissenso ideologico fra i marxisti e il sionismo era emerso con forza, come aveva mostrato la guerra dei Sei giorni. Da allora il Pci si era schierato apertamente con i paesi arabi, seguendo la politica dell'Urss che aveva rotto le relazioni diplomatiche con Israele, mentre i socialisti e i repubblicani erano rimasti fedeli allo Stato ebraico. A suo avviso ne erano derivati: una vera e propria fuga di molti ebrei dal Pci verso il Psi, la nascita di nuove realtà grazie alla partecipazione di giovani ebrei al movimento studentesco, ma anche l'allontanamento di molti ebrei di sinistra dalle posizioni di Israele ${ }^{22}$. Soltanto la crisi del comunismo, notava Molinari, aveva dato il via ad una fase nuova e più distesa, della relazione fra la sinistra italiana e gli ebrei.

Fino ai primi anni Novanta, dunque, nessuno aveva studiato le relazioni fra la sinistra e la questione ebraica interrogando la cultura politica di matrice marxista, soffermandosi sull'antisemitismo e su come socialisti e comunisti l'avevano rappresentato. La crisi del comunismo, la trasformazione della società italiana, il mutare dei partiti politici di sinistra e il modificarsi del conflitto in Medio Oriente aprirono scenari diversi e portarono gli storici a porsi nuove domande.

\section{La sinistra italiana e il conflitto arabo-israeliano}

Da allora, la storiografia italiana che si è occupata di questi argomenti ha approfondito due questioni principali: 1) il filone maggioritario ha studiato le reazioni della sinistra di fronte al conflitto mediorientale e alla politica di Israele; 2) mentre quello minoritario ha ricostruito le riflessioni degli esponenti del socialismo italiano sulla questione ebraica e ha analizzato come i partiti e i movimenti di sinistra hanno rappresentato le persecuzioni antiebraiche del Novecento.

Il rapporto fra il Pci e Israele ha trovato ampio spazio nei lavori di Luca Riccardi, autore di due monografie su questo tema ${ }^{23}$. Sono libri che rientrano a pieno titolo nella storiografia delle relazioni internazionali, in cui Riccardi con un'analisi dettagliata delle carte del partito, delle fonti parlamentari e di quelle a stampa ripercorre le evoluzioni della politica del Pci dal

${ }^{22}$ M. Molinari, La sinistra e gli ebrei in Italia. 1967-1993, cit.

${ }^{23}$ L. Riccardi, Il «Problema Israele», cit.; Id., L'internazionalismo difficile, cit. Cfr. anche G. Santese, "Il Partito comunista italiano e la questione palestinese (1945-1956)", cit.; D. Caviglia, "La politica dell'Italia e il conflitto arabo-israeliano. L'atteggiamento italiano nella documentazione diplomatica francese", Nuova storia contemporanea, 1, 2005, pp. 17-50. 
1948 al crollo del comunismo. Nel primo volume, che copre 1'arco cronologico 1948-1973, mostra come dal sostegno espresso nel 1948, i comunisti passarono velocemente a posizioni di dura critica, determinate dagli sviluppi della politica mediorientale dell'Unione Sovietica e culminate con la guerra dello Yom Kippur. Nel secondo volume, Riccardi porta l'analisi fino alla caduta del muro di Berlino ed estende lo sguardo all'intera politica mediorientale, sottolineando i limiti dei comunisti italiani convinti sostenitori del panarabismo, come strumento dell'anti-imperialismo, e poi in difficoltà nel cogliere alcuni passaggi degli anni Settanta e Ottanta. In particolare, nelle settecento pagine del secondo libro, Riccardi analizza la politica del partito guidato da Berlinguer che offrì un importante contributo alla sensibilizzazione dell'opinione pubblica italiana alla causa dell'Olp: un'azione che, secondo l'autore, indusse il Pci a errori di valutazione. Avendo condannato la svolta filoamericana di Sadat, dopo la guerra del Kippur del 1973, i comunisti si trovarono d'accordo con Arafat nel criticare gli accordi di Camp David del 1978. Di fatto, ai loro occhi Israele - spiega Riccardi continuò a lungo a essere considerato l'avamposto dell'imperialismo: uno Stato con mire aggressive, costruito sulla logica dell'espansione territoriale. Solo dai primi anni Ottanta, gli esponenti del Pci riuscirono a scindere le responsabilità del governo di Tel Aviv da quelle del popolo israeliano e negli anni successivi ad inaugurare una nuova politica che ebbe in Achille Occhetto e Piero Fassino i suoi sostenitori più convinti.

Anche Claudio Brillanti si è confrontato con il conflitto mediorientale analizzando la reazione dell'intera sinistra italiana nel periodo 1948-1973. $\mathrm{Ne}$ è emersa una ricerca scrupolosa che prende in esame fonti a stampa, atti parlamentari e documenti di archivio e testimonia la presenza di diverse posizioni. In realtà, in questo lavoro, che comprende i marxisti più radicali, il Pci, il Psi, i socialdemocratici, la cultura azionista, i repubblicani e la sinistra democristiana non è chiara la definizione di sinistra illustrata dall'autore nell'introduzione, mettendo insieme esperienze, culture, identità politiche che poco ebbero in comune. Di fatto, a parte la destra DC, i liberali e il Msi, il volume si occupa dei protagonisti dell'intera politica italiana in anni decisivi per la sua trasformazione, ricostruendo i conflitti all'interno delle formazioni politiche e le differenze fra un partito e l'altro ${ }^{24}$. Valentino Baldacci, invece, ha pubblicato un lavoro in cui ha scandagliato le fonti a stampa per ricostruire una vera e propria mappa quotidiana della reazione dei principali partiti della sinistra italiana di fronte alla guerra dei Sei giorni, quando maturò un durissimo scontro fra il Pci e il Partito socialista uni-

\footnotetext{
${ }^{24} \mathrm{C}$. Brillanti, Le sinistre italiane e il conflitto arabo-israelo-palestinese, cit.
} 
ficato e Israele venne descritto da molti esponenti della tradizione marxista come un paese espansionista, militarista, violento e razzista ${ }^{25}$.

Gli autori di questi volumi, diversi per l'arco cronologico considerato e per i soggetti politici presi in esame, convergono sui risultati ed evidenziano che il Pci è stato vicino a Israele soltanto nei primi momenti dalla sua nascita; ha assunto una posizione radicalmente filo-araba - e poi filo-palestinese - sin dai primi anni Cinquanta e smussato il proprio antisionismo solo alla metà degli anni Ottanta. Il Psi, invece, ha seguito la politica del Pci fino ai primi anni Sessanta, con Nenni ha espresso una politica filo-israeliana, smussata dalla segreteria De Martino e in seguito, durante l'era Craxi, è diventato un paladino della causa palestinese. I socialdemocratici e i repubblicani, piccoli partiti rispetto alle grandi formazioni di massa, hanno manifestato il loro sostegno a Israele durante le diverse fasi della loro storia.

In questi libri sul conflitto mediorientale, la politica sembra il frutto delle scelte e delle azioni e non l'espressione delle identità culturali, che vengono enunciate, ma non analizzate. Riccardi nomina spesso nel suo secondo volume «l'ideologia comunista», Brillanti sottolinea la differenza fra la cultura laica e socialdemocratica e quella del Pci di fronte alle questioni in gioco e Baldacci evidenzia la profonda distanza fra il Psi di Nenni e il Pci di Longo. Cosa intendono questi autori per ideologia? E qual è il rapporto fra le ragioni della Guerra Fredda e le identità culturali delle forze politiche in campo davanti al conflitto in Medio Oriente? Il Pci fu antisionista perché era fedele all'Urss o perché era comunista? E cosa è più rilevante nell'assunzione delle decisioni politiche, la cultura di provenienza, l'identità culturale o la contingenza dei diversi contesti?

Nel 1951 Federico Chabod suggerì un metodo per rispondere a domande come queste e scrisse che comprendere la politica significa analizzare i rapporti fra i fatti e le idee, fra le aspirazioni e i risultati ottenuti. Egli dubitava dell'esistenza dei cosiddetti «"interessi permanenti”», quella

sorta di divinità ascosa che dovrebbe star al disopra di tutto quanto costituisce la vita concreta di un popolo, lotte politiche, ideali e ideologie, cozzar di passioni, per costituire il presupposto e lo scopo della politica estera, la stella polare a cui tener l'occhio fisso durante la navigazione perigliosa, senza curar il resto ${ }^{26}$.

Seguendo l'insegnamento del grande storico valdostano, immaginiamo che dichiarare l'esistenza di ideologie, senza analizzarle nel loro dispiegarsi, negli scritti dei teorici e degli intellettuali, sottovalutando la presenza di

${ }^{25}$ V. Baldacci, 1967. Comunisti e socialisti di fronte alla Guerra dei Sei giorni, cit.

${ }^{26}$ F. Chabod, Storia della politica estera italiana dal 1870 al 1896, vol. I, Laterza, Bari, 1971, p. 11. 
culture politiche, idee, concetti, immagini, stereotipi, limitando l'analisi alle dichiarazioni sulla stampa o a quelle degli organi di partito, non faciliti la comprensione del problema. E, in effetti, l'analisi del rapporto fra la sinistra e il conflitto arabo-israeliano, disgiunta dalla riflessione sulla questione ebraica, non appare sufficiente a spiegare né l'uno né l'altro dei termini del problema; anche perché, se ci fermassimo alla reazione dei partiti di sinistra di fronte alla politica di Israele, non avremmo molto da aggiungere a ciò che già sappiamo e cioè che dal 1948 a oggi non sono stati equidistanti, in linea con il tradizionale filo-arabismo della politica estera italiana.

Lo hanno dimostrato, a metà degli anni Ottanta, due sociologi con una ricerca sulla guerra del Libano $^{27}$. Roberta Cipollini e Enzo Campelli hanno introdotto temi ripresi da molti autori negli anni Duemila ricordando che in tutta la stampa italiana, e in particolare in quella di sinistra, dopo la tragedia dei campi di Sabra e Chatila, si diffusero due immagini: quella degli ebrei come nuovi nazisti e quella del popolo biblico, protagonista di una storia di sopraffazione e violenza, seguace di un Dio terribile e violento ${ }^{28}$. Descritti attraverso categorie metastoriche, schiacciati sulla politica israeliana, gli ebrei furono percepiti, e rappresentati, come dotati di una coesione e una potenza capaci di resistere a secoli di atroci persecuzioni; come se la loro religione, a differenza di altre, fosse in grado di renderli diversi e non assimilabili ${ }^{29}$. E in effetti, come ha notato Roberta Cipollini, alla base dei giudizi politici sulle vicende mediorientali, vi fu una valutazione sulla condizione ebraica considerata come la premessa della politica estera di Israele. In realtà, ricerche successive hanno mostrato che il confronto fra gli israeliani e i nazisti fu utilizzato dalla sinistra ben prima del 1982, anche se negli anni Ottanta ebbe ampia diffusione, come hanno mostrato Arturo Marzano e Guri Schwarz ${ }^{30}$.

I due storici hanno ricostruito la reazione dell'opinione pubblica di fronte alla guerra del Libano e all'attentato alla Sinagoga di Roma dell'ottobre del 1982, considerandolo un evento rivelatore di importanti tendenze della storia della questione arabo-israeliana ${ }^{31}$. Confermando un giudizio presente in alcune ricerche precedenti, hanno mostrato come dal 1967 la politica italiana si sia gradualmente spostata verso la causa palestinese condividendo, 1984.

${ }^{27}$ E. Campelli, R. Cipollini (a cura di), Contro il seme di Abramo, FrancoAngeli, Milano,

${ }^{28}$ Ibid., p. 104.

${ }^{29}$ Ibid., p. 120.

${ }^{30} \mathrm{C}$. Brillanti, Le sinistre italiane e il conflitto arabo-israelo-palestinese, cit.; A. Tarquini, "1968. Un anno dalla guerra dei Sei giorni, venti dalla nascita di Israele", cit., pp. 67-88.

${ }^{31}$ A. Marzano, G. Schwarz, Attentato alla Sinagoga. Roma, 9 ottobre 1982. Il conflitto israelo-palestinese e l'Italia, Viella, Roma, 2013. 
nella maggioranza dei casi, le posizioni che fino a quel momento erano state del $\mathrm{Pci}^{32}$. A sostegno di questa interpretazione, Marzano e Schwarz non limitano l'analisi alla stampa di partito ma, meritoriamente, la estendono all'editoria, dai fumetti ai pamphlets dei gruppi studenteschi, ragionando quindi sugli stereotipi, sui modi di rappresentare Israele o di presentare i palestinesi descritti come un simbolo della lotta anti-imperialista e accomunati ai Vietcong, quando non ai partigiani antifascisti del $1943^{33}$. A questo proposito ricordano che «il movimento studentesco aveva chiara convinzione che esistesse un legame unico tra le forze che nei vari contesti sostenevano l'imperialismo americano» ${ }^{34}$. In effetti, la difesa della causa palestinese non rimase confinata ad un problema di politica internazionale: per tutti gli anni Settanta, e poi in modo evidente dopo l'invasione del Libano, molti protagonisti del dibattito pubblico, e moltissimi esponenti della sinistra extraparlamentare, diffusero un'immagine che avrebbe avuto ampio successo: quella degli israeliani come nuovi nazisti, già analizzata nel volume di Cipollini e Campelli ${ }^{35}$. Da Servire il popolo a Lotta continua, per arrivare ad alcune firme della stampa vicina al Pci, intellettuali e politici sostennero che gli ebrei imponevano ai palestinesi le sofferenze subite durante il regime nazista. Non stupisce, dunque, che il clima politico alla vigilia dell'attentato alla sinagoga di Roma del 1982 fosse ostile nei confronti dello Stato ebraico. Ovviamente questo non significa che la presenza di stereotipi antisemiti nel dibattito pubblico italiano abbia determinato l'attentato terroristico compiuto da un commando palestinese il 9 ottobre 1982, come ricordano, a più riprese, Marzano e Schwarz.

A questo proposito, i due autori notano che sebbene antisemitismo e antisionismo siano realtà profondamente diverse, i critici di Israele assunsero toni e utilizzarono linguaggi di antica matrice antisemita tanto da rendere difficile una vera e propria distinzione fra i due livelli del discorso ${ }^{36}$. Marzano e Schwarz descrivono un clima nel quale si fa largo uso di immagini e giudizi antisemiti, ma non analizzano i soggetti politici che li esprimono, offrendo un'immagine della cultura politica italiana degli anni Ottanta, a volte eccessivamente compatta. Per esempio, non dialogando con autori come George Mosse o Enzo Traverso - ma del resto nessuno degli autori

\footnotetext{
${ }^{32}$ Ibid., pp. 52-32.

${ }^{33}$ Ibid., pp. $73-85$.

${ }^{34}$ Ibid., p. 79.

35 Ibid., pp. 88-94.

${ }^{36}$ Anche Marta Brachini ha ricordato che dopo la guerra del Libano nella stampa di sinistra si diffuse l'immagine di un'inversione per cui i perseguitati di ieri scaricano su un altro popolo le stesse violenze che hanno subito. Cfr. M. Brachini, A. Maori, Ebraismo. Ricostruire dalle macerie, Stampa alternativa, Roma, 2013, pp. 69-111.
} 
italiani citati in questo saggio ha sentito l'esigenza di farlo - sostengono che la sinistra ha ereditato pregiudizi e stereotipi di matrice cristiana, come se non esistesse un antisemitismo di sinistra, autonomo dall'antica cultura antigiudaica e, soprattutto, come se la critica contro Israele e il sionismo fosse emersa nel 1982, o al massimo iniziata durante la guerra dei Sei giorni, scelta nel libro come evento periodizzante. Enfatizzare la rottura del 1967 significa: da un lato responsabilizzare Israele che, come tutti notano, divenne un paese aggressore e non più una vittima degli arabi, e dall'altro sottovalutare il fatto che l'esistenza di uno Stato ebraico ha rappresentato un problema per la sinistra di tutto il mondo dalla nascita del sionismo, e non dal 1967, così come l'emersione di stereotipi antisemiti nel dibattito pubblico non è una novità degli anni Ottanta. George Mosse, nel 1971, studiando i rapporti fra la socialdemocrazia e la questione ebraica durante la Repubblica di Weimar partì da Karl Kautsky mostrando come il teorico del socialismo, e l'intero suo partito, svilupparono una profonda distanza dalla questione ebraica e dalle ragioni del sionismo. Dunque, per essere radicalmente antisionisti fino a lambire l'antisemitismo, come accadde a molti esponenti della sinistra italiana, non è necessario veicolare pregiudizi di matrice cristiana. La sinistra, lo ha spiegato chiaramente Enzo Traverso, ha espresso sin dalla fine del XIX secolo le sue resistenze culturali all'accoglienza della questione ebraica che precedono di cento anni la guerra dei Sei giorni e quella del Libano ${ }^{37}$.

E, allora, per comprendere il problema occorre partire dalle identità politiche e culturali del soggetto di cui stiamo parlando e chiedersi se e quale tipo di rapporto questo o questi gruppi politici hanno avuto con gli ebrei nei centocinquanta anni della loro storia.

\section{La sinistra italiana, il sionismo e l'antisemitismo}

Dalla fine degli anni Ottanta, accanto agli studi sul conflitto araboisraeliano, sono stati pubblicati i primi saggi sulla relazione fra la sinistra e gli ebrei. Furio Biagini, ad esempio, ha analizzato la riflessione dei teorici

\footnotetext{
${ }^{37}$ E. Traverso, Les marxistes et la question juive, cit., Per una riflessione sugli storici italiani cfr. A. Donno, "La storia di Israele in Italia", in Israele mezzo secolo, Quaderni di nuova storia contemporanea, Luni, Milano, 1998, pp. 169-181. Secondo Donno, solo alla fine degli anni Ottanta il crollo del comunismo privò «i professionisti della polemica contro Israele» di un sostrato ideologico e inaugurò una fase nuova. In effetti, la scarsa autonomia dalle logiche prodotte dalla Guerra Fredda può spiegare la mancanza di studi italiani su Israele. D'altra parte, il non essere comunista non è sufficiente a garantire analisi approfondite.
} 
del movimento operaio, sostenitori di un marxismo scolastico, nato nella cultura positivistica e progressista della Seconda Internazionale, incapace, a suo avviso, di cogliere la natura dell'antisemitismo moderno. A questo proposito ha sottolineato che i marxisti di fine Ottocento «condividevano l'opinione dei loro contemporanei liberali, secondo i quali il progresso culturale, economico e sociale avrebbe avuto ragione del particolarismo nazionale e spinto la società umana sulla strada dell'internazionalismo» ${ }^{38}$. Coerentemente con queste premesse erano antisionisti, consideravano l'antisemitismo una reazione della piccola borghesia contro l'avvento della modernità e pensavano che gli ebrei avessero due possibilità: combattere con i proletari di tutto il mondo per costruire un mondo diverso, oppure unirsi agli altri ebrei nella fondazione di un grande ghetto mondiale ${ }^{39}$. In questo panorama, come ha sottolineato Alberto Cavaglion, il caso di Felice Momigliano, inviato dell'Avanti! ai congressi sionisti, fu un'eccezione nel Psi di fine secolo. Ebreo, studioso di storia delle religioni e attento alle ragioni di un socialismo non classista, Momigliano descrisse il nuovo movimento politico con sguardo attento e animo empatico.

Come si può notare, nelle prime ricostruzioni, gli storici hanno privilegiato una fase della storia dei rapporti fra la sinistra italiana e la questione ebraica - concentrandosi prevalentemente sul socialismo di fine secolo - e si sono impegnati in ricerche su temi circoscritti. In questo scenario, il volume curato nel 2007 da Mario Toscano rappresenta una novità perché copre un arco cronologico molto ampio e coniuga la storia delle relazioni internazionali con quella delle culture politiche e quella degli ebrei tenendo insieme questi diversi argomenti, tradizionalmente assegnati ad ambiti di ricerca separati distinti ${ }^{40}$. Gli autori hanno condotto una ricerca di lungo periodo, dalla fine del XIX secolo fino agli anni Settanta del Novecento, ricostruendo come il Psi ha interpretato e rappresentato ai suoi militanti, all'elettorato e all'opinione pubblica italiana il sionismo, l'antisemitismo e, dopo il 1948, il conflitto arabo-israeliano ${ }^{41}$.

Possiamo sintetizzare gli aspetti più rilevanti del lavoro isolando alcune grandi questioni. Analogamente a ciò che accadde nel resto d'Europa, anche in Italia, dopo le iniziali incertezze sull'atteggiamento da tenere di fron-

${ }^{38}$ F. Biagini, "Sionismo, marxismo e socialismo italiano (1897-1917)", cit., p. 323.

${ }^{39}$ Cfr. G. Tortorelli, "L'Affaire Dreyfus e i Socialisti italiani”, cit., pp. 498-513. A. Cavaglion, "Il sionismo nella stampa socialista di fine Ottocento. Osservazioni preliminari", cit., pp. 223-236.

${ }^{40}$ Cfr. M. Toscano (a cura di), Ebraismo, sionismo e antisemitismo nella stampa socialista, cit. Anche se non direttamente dedicato al rapporto fra ebrei e sinistra, cfr. L. Mangoni, "Ebraismo e antifascismo", Studi storici, 1, 2006, pp. 65-78.

${ }^{41}$ M. Toscano, "Introduzione: i confini delle identità", in Id. (a cura di), Ebraismo, sionismo e antisemitismo nella stampa socialista, cit., p. 8. 
te alla vicenda del capitano Dreyfus, i socialisti dedicarono grande attenzione all'evento, descrivendolo come un momento dello scontro tra la reazione clericale e le forze che difendevano la libertà, facendo fatica a cogliere i contenuti dell'antisemitismo, rimanendo ancorati a una visione stereotipata dell'ebraismo e degli ebrei, ricchi banchieri o perseguitati, che non sarebbe venuta meno negli anni successivi ${ }^{42}$. Analizzando le differenti posizioni di riformisti e massimalisti di fronte all'antisemitismo del regime fascista emerge un dato importante e cioè che mentre i primi si mostrarono relativamente sensibili al problema della persecuzione degli ebrei, i secondi considerarono l'antisemitismo un «fatto strutturale», espressione del capitalismo, non diverso da altre manifestazioni di violenza espresse dal regime fascista $^{43}$. A questo proposito Luca La Rovere ha scritto che la sostanziale incapacità di cogliere la portata e le conseguenze della campagna antiebraica del fascismo deve essere attribuita alla spiccata tendenza a leggere in chiave ideologica la realtà e, dunque, a un vero e proprio deficit culturale ${ }^{44}$. Questo atteggiamento cambiò parzialmente dopo la seconda guerra mondiale quando la stampa socialista, come quella antifascista in genere, si soffermò su quanto accaduto nei campi d'internamento, senza riguardo per l'appartenenza nazionale o religiosa ${ }^{45}$. Dal 1945 alla fine degli anni Sessanta, il Psi ebbe un atteggiamento ambiguo e oscillante anche nei confronti del sionismo e di Israele. Nel 1948 accolse con grande entusiasmo la nascita del nuovo Stato ${ }^{46}$. Già all'inizio degli anni Cinquanta, tuttavia, cambiò posizione e assunse un atteggiamento meno aperto, accettando e diffondendo la versione sovietica della congiura dei medici e del processo Slansky, denigrando il sionismo e negando l'esistenza di forme di antisemitismo nei paesi del blocco socialista ${ }^{47}$. Mostrando di credere nel valore della memoria del genocidio degli ebrei, sin dai primi anni del dopoguerra, i socialisti presentarono le testimonianze dei sopravvissuti come una parte essenziale del tessuto culturale e politico dei paesi democratici. Questa operazione non venne accompagnata da una riflessione sulle ragioni per cui il nazismo e il fascismo avevano fatto dell'antisemitismo uno dei tratti caratteristici della propria politica totalitaria: nel dedicare così tanto spazio ai campi di con-

42 F. Del Regno, "L'antisemitismo e il sionismo nelle cronache e nelle analisi dell'Avanti! (1897-1920)", ibid., pp. 30-31, 34-35.

${ }^{43}$ L. La Rovere, "Fascismo, 'questione ebraica' e antisemitismo nella stampa socialista. Un'analisi di lungo periodo: 1922-1967”, ibid., p. 101.

${ }^{44}$ Ibid., p. 102.

${ }^{45}$ Ibid., p. 119.

${ }^{46}$ A. Tarquini, "Il partito socialista fra guerra fredda e 'questione ebraica': sionismo, antisemitismo e conflitto arabo-israeliano nella stampa socialista dalla nascita della Repubblica alla fine degli anni Sessanta", ibid., p. 165.

${ }^{47}$ Ibid., pp. 186-187. 
centramento, i socialisti mostrarono di considerare il totalitarismo come una nuova forma di barbarie e di considerare più importante la denuncia dell'orrore rispetto alla riflessione sulle origini e sulle manifestazioni della violenza contro gli ebrei ${ }^{48}$. In effetti, dalla stampa socialista di quel periodo sembra emergere un'ovvia identificazione fra nazismo e antisemitismo da un lato e democrazia e lotta all'antisemitismo dall'altro, come se l'antisemitismo non fosse stato un aspetto della cultura europea, non avesse avuto una sua storia, ma fosse invece, uno dei caratteri del nazismo e del fascismo, quindi fosse proprio e peculiare di regimi barbari, sconfitti dall'avvento della democrazia. Un esempio di questa tendenza è nel commento pubblicato dall'Avanti! al diario di Anna Frank, uscito in italiano per Einaudi nell'aprile del 1954. La maturità di Anna, le sue idee sulla vita, la sua speranza e la sua fiducia nell'umanità furono i temi della recensione di Giuseppe Petronio, che non ebbe niente da dire sul fatto che Anna Frank era una giovane ebrea ${ }^{49}$.

Dall'inizio degli anni Sessanta, in concomitanza con i primi passi del centro-sinistra e dunque con una politica meno schiacciata sullo storico alleato comunista, i socialisti italiani mostrarono maggiore attenzione sia nei confronti di Israele, sia rispetto all'antisemitismo e alla Shoah. A testimoniare questo mutato atteggiamento vi furono: lo spazio che i giornali socialisti dedicarono alla realtà della minoranza ebraica in Unione Sovietica; la riflessione che la stampa del Psi propose sulla persecuzione degli ebrei durante il nazismo e la seconda guerra mondiale; una diversa posizione politica rispetto al conflitto arabo-israeliano per cui nella guerra del giugno 1967 i socialisti si schierarono decisamente a favore di Israele, contestando la politica estera dell'Urss; il duro scontro che ebbero con i comunisti, schierati con Nasser; e infine, la discussione che il Psi sollevò all'interno della compagine governativa di centro-sinistra esprimendo un orientamento diverso da quello della sinistra democristiana ${ }^{50}$.

Anche Matteo Di Figlia, nel suo Israele e la sinistra, analizza il tema nella sua interezza ripercorrendo i percorsi biografici di alcuni ebrei italiani che, a diverso titolo, aderirono a movimenti o partiti di sinistra dal 1945 a giorni più recenti ${ }^{51}$ : si tratta di autorevoli protagonisti della cultura e della politica come Tullia e Bruno Zevi, Emilio Sereni, Franco Fortini,

${ }^{48}$ Ibid., pp. 184-185.

${ }^{49}$ Ibid.

${ }^{50}$ Ibid., p. 197.

${ }^{51}$ M. Di Figlia, Israele e la sinistra, cit.; cfr. anche F. Nicolucci, Sinistra e Israele. La frontiera morale dell'Occidente, Salerno editrice, Roma, 2013. Ricco di stimoli interessanti, il libro non è un saggio storiografico, ma il frutto di una riflessione sulla decadenza dell'Occidente e sugli attuali rapporti fra la sinistra e lo Stato ebraico. 
Amos Luzzatto, Luca Zevi e Fiamma Nirenstein, per nominarne soltanto alcuni. Sin dalle prime pagine, le ragioni di questa scelta non convincono completamente. Gli ebrei, come gli appartenenti ad una qualsiasi religione, nazione, cultura, etnia, non sono necessariamente di destra o di sinistra e, in effetti, non è del tutto chiaro perché l'autore dia per scontata una vicinanza politica, mostrando l'evoluzione, senza dubbio interessante, di questi noti personaggi. Per dichiarare che esiste un legame privilegiato fra la sinistra e gli ebrei? È una proposta interpretativa che dovrebbe essere dimostrata, soprattutto pensando che la maggioranza degli ebrei, come degli altri italiani, aderì al regime fascista. In ogni caso, è sufficiente essere celebri per rappresentare la categoria degli ebrei di sinistra? Convinto che «non ci fu un'automatica correlazione tra critica a Israele e ortodossia comunista, né tra quest'ultima e l'antisemitismo di sinistra ${ }^{52}$, Di Figlia sostiene l'esistenza di «legami ideologici tra tutte le sinistre italiane e Israele che risentirono della contrapposizione fra i blocchi, ma che non si recisero» negli anni della Guerra Fredda ${ }^{53}$. In realtà, non analizza questi legami, non si occupa dei rapporti fra Israele e i partiti di sinistra, né di antisemitismo, e si sofferma su alcune esperienze dell'ebraismo italiano senza spiegare perché dovrebbero essere rappresentative di un universo così complesso. Il volume racconta le vicende familiari e personali di alcuni ebrei di sinistra attraverso testimonianze e interviste. In questo senso, ad esempio, Di Figlia ricostruisce l'esperienza dei centri giovanili HeChalutz, di orientamento socialista, e quella delle nuove generazioni che, soprattutto dall'inizio degli anni Ottanta, furono impegnate nella discussione sulla propria identità, desiderose di superare le contrapposizioni ideologiche che avevano segnato la vita delle generazioni precedenti.

E, infine, ricordiamo i contributi recenti di maggiore rilievo: quello del 2007 di Gadi Luzzatto Voghera, Antisemitismo a sinistra, e quello di Michele Battini del 2010 Il socialismo degli imbecilli. Il piccolo volume di Luzzatto Voghera contiene alcune osservazioni particolarmente importanti per chi voglia studiare questi argomenti. Ad esempio nell'introduzione si legge:

dire che l'antisemitismo e la sinistra sono due concetti incompatibili è storicamente sbagliato e politicamente ipocrita. [...] è un problema storiografico e teoretico il fatto che alcune correnti fondative del progressismo europeo contenessero anche discorsi apertamente antiebraici ${ }^{54}$.

\footnotetext{
${ }^{52}$ M. Di Figlia, Israele e la sinistra, cit., p. 5.

${ }^{53}$ Ibid., p. 24.

${ }^{54}$ G. Luzzatto Voghera, Antisemitismo a sinistra, Einaudi, Torino, 2007, p. XI.
} 
Luzzatto Voghera ricorda che l'antisemitismo moderno nacque dall'identificazione degli ebrei con la borghesia improduttiva legata al denaro: un'immagine presente nelle opere di Marx e di molti socialisti utopisti. A questo proposito, sostiene che quando gli studiosi affrontano il saggio giovanile Sulla questione ebraica ancora oggi evitano di considerare gli ebrei come il tema centrale dello scritto e «si esercitano nell'indicarne le radici post-feuerbachiane e nel collocare l'operetta fra i primi esempi di una più ampia critica dello Stato ${ }^{55}$. E, invece, come nota l'autore, l'importanza del lavoro di Marx sta nella sua diffusione.

Il testo era probabilmente letto assieme agli altri testi di Marx nelle società di mutuo soccorso e nelle case del popolo, dove non molti sapevano collocare Hegel o Feuerbach mentre in genere avevano piuttosto ben presente chi erano gli ebrei: in tal modo l'antisemitismo, nella sua versione di identificazione dell'ebreo con il denaro e il potere, lungi dal rimanere una sovrastruttura divenne una componente significativa pronta a riemergere in diverse occasioni ${ }^{56}$.

Luzzatto Voghera ricorda che soltanto dopo la prima guerra mondiale, l'Internazionale socialista prese atto dell'urgenza della questione ebraica, salvo modificare nuovamente questo atteggiamento dopo l'esperienza della Shoah e della seconda guerra mondiale. Dall'inizio degli anni Cinquanta, infatti, la posizione egemonica di Mosca sulla sinistra internazionale impedì che socialisti e comunisti prendessero atto della presenza di pratiche violente e discriminatorie contro gli ebrei in Urss. Da allora, la sinistra ha continuato a descrivere gli ebrei facendo uso di astrazioni, di stereotipi, di descrizioni caricaturali che poco hanno a che fare con la realtà: di nuovo ricchi banchieri o proletari poverissimi ${ }^{57}$. Secondo Luzzatto Voghera, dopo l'immagine dell'ebreo capitalista e quella della vittima del nazifascismo, negli ultimi trenta anni, e in modo evidente dalla guerra del Libano, la sinistra ha spesso e volentieri identificato gli ebrei con i sionisti dichiarandosi antisionista e difendendosi dall'accusa di antisemitismo. Questo è stato possibile grazie ad uno slittamento semantico della definizione di sionismo, non più considerato come un movimento di liberazione e rinascita nazionale, ma come uno strumento economico militare nelle mani del capitalismo imperialistico, con il risultato di creare una «meta-realtà nella quale l'unico vero comune denominatore è il linguaggio antisemita e l'ossessiva reiterazione dei più vieti stereotipi proposti da questo nei secoli»» ${ }^{58}$. Dunque, non

\footnotetext{
${ }^{55}$ Ibid., p. 30.

${ }^{56}$ Ibid.

${ }^{57}$ Ibid., p. 35.

${ }^{58}$ Ibid., p. 75.
} 
solo è esistito un antisemitismo di sinistra ma, come mostra efficacemente Luzzatto Voghera, ha una tradizione antica. Si potrebbe obiettare che la storia dei socialisti e quella dei comunisti non coincidono, o almeno non sempre. Lo dimostra il fatto che alcuni socialisti, e soprattutto i socialdemocratici, nel dopoguerra hanno assunto una posizione di dura critica nei confronti dell' antisemitismo e di quanto accadeva in Urss a partire dagli anni Cinquanta, sostenuti dalle battaglie condotte dall'Internazionale socialista. In ogni caso, la sostanza del discorso di Luzzatto Voghera non cambierebbe.

Michele Battini ha sostenuto una tesi molto diversa analizzando il più importante pregiudizio antisemita dell'età contemporanea: quello, molto noto, e per questo studiato anche da autorevoli storici, secondo il quale gli ebrei sarebbero il simbolo del capitalismo ${ }^{59}$. Si tratta di un paradigma che ha attraversato svariate culture politiche - la dottrina sociale cattolica, l'economia neocristiana, i gruppi del socialismo premarxista, il sindacalismo rivoluzionario, il sindacalismo nazionale e poi il fascismo - e quindi raggiunto personaggi assai diversi: cristiani, nazionalisti, intellettuali di destra ed esponenti del movimento operaio. Convinto dell'esistenza di una connessione fra la tradizione antigiudaica cristiana e l'ideologia antisemita, Battini mostra come questo antisemitismo moderno, dal carattere transpolitico, avesse una forte connotazione economica e sociale e fosse il veicolo di una protesta contro la modernità e i cambiamenti che questa aveva imposto alla società europea ${ }^{60}$.

Con un'interpretazione che deve molto agli studi di Zeev Sternhell, Battini sostiene che l'antisemitismo di sinistra non derivò dal patrimonio ideologico del socialismo marxista ma da «quello della letteratura socialista francese del primo Ottocento e dei testi di Leroux, Fourier, Toussenel $\rangle^{61}$. Coerentemente con questo presupposto, egli non analizza La Questione ebraica di Marx, che utilizzò gli stessi stereotipi presenti nella cultura europea di metà Ottocento e definì gli ebrei il simbolo del denaro ${ }^{62}$. A suo avviso, anche i più noti socialisti antisemiti, come Fourier, Proudhon e Toussenel, mutuarono le loro riflessioni sugli ebrei da una letteratura di matrice cristiana. «L'ipotesi che io suggerisco - scrive a questo proposito - è che il testo di Toussenel, vera pietra miliare del socialismo antisemita, sia fortemente debitore dell'apologetica sociale della religione cattolica ${ }^{63}$.

${ }^{59}$ M. Battini, Il socialismo degli imbecilli. Propaganda, falsificazione, persecuzione degli ebrei, Bollati Boringhieri, Torino, 2010.

${ }^{60}$ Ibid., pp. 35-86.

${ }^{61}$ Ibid., p. XIII.

${ }^{62}$ Ibid., p. X.

${ }^{63}$ Ibid., p. 49. 
Ribadendo una proposta interpretativa secondo cui le persecuzioni antiebraiche non sarebbero che una riedizione moderna dell'antigiudaismo cristiano, Battini non pensa che esista un problema legato alla nazionalizzazione degli ebrei e al loro rapporto con l'universalismo della sinistra. Le poche o tante volte in cui la sinistra fu antisemita, mutuò dal pensiero cristiano il suo antigiudaismo antimoderno. Senza tenere conto delle riflessioni di Annie Kriegel, e soprattutto di quelle di George Mosse o di Enzo Traverso, e dei tanti che si sono interrogati sulla modernità dell'antisemitismo, e sulla presenza di un antisemitismo di sinistra, abbiamo qui un quadro molto diverso, tutto concentrato sul tema dell'anticapitalismo e sulla sua presunta antimodernità che si estenderebbe al fascismo. Potremmo sottolineare che ormai da molti anni il fascismo viene studiato dagli storici come un regime moderno o notare che le forme dell'anticapitalismo non sono necessariamente un'espressione della critica contro la modernità. Potremmo, per esempio, discutere su quanto il comunismo abbia rappresentato una severa critica della modernità capitalistica $\mathrm{e}$ al contempo si sia presentato $\mathrm{al}$ mondo come l'espressione più autorevole dell'emancipazione dell'uomo da uno stato di subalternità e di schiavitù, ma questo non sarebbe argomento del presente saggio e ci porta, dunque, ad alcune conclusioni ${ }^{64}$.

\section{Conclusioni}

Come si è visto, in Italia la storia dei rapporti fra la sinistra e gli ebrei ha suscitato l'interesse degli storici soltanto negli anni Novanta. Fino a quel momento se ne erano occupati politici e giornalisti impegnati nella discussione sugli equilibri geopolitici provocati dalla Guerra Fredda. Dal tramonto del comunismo, come ordine e visione del mondo, e dalla crisi dei partiti della cosiddetta Prima repubblica, si è andata formando una storiografia che si può dividere in due campi: alcuni hanno studiato i rapporti fra la sinistra e Israele, mentre altri hanno analizzato il pensiero dei socialisti di fronte

${ }^{64} \mathrm{M}$. Consonni, L'eclisse dell'antifascismo. Resistenza, questione ebraica e cultura politica in Italia dal 1943 al 1989, Laterza, Roma-Bari, 2015. Colpisce per la tesi che lo sorregge: «il rapporto identitario fra Resistenza e deportazione era stato veramente vissuto e percepito come una forma di lotta partigiana al nazifascismo. [...] La necessità del ricordo e il dovere della testimonianza erano imposti dall'urgenza di combattere la tendenza revisionista» (p. 258). Ne emerge un quadro per cui la battaglia antifascista e quella contro l'antisemitismo si identificano, che è esattamente il contrario di quanto sostengono autorevoli studiosi. Così, l'autrice giunge ad un epilogo tanto discutibile quanto perentorio nel quale afferma: «In tale discorso lo sterminio ebraico è rimasto da solo a difendere la storia e la memoria dell'antifascismo, garante estremo della democrazia» (p. 302). 
alla questione ebraica, sottolineando le rappresentazioni che la sinistra ha proposto dell'antisemitismo moderno.

Come abbiamo visto, nell'ambito del primo filone di studi, sono stati pubblicati diversi saggi che hanno ampliato le nostre conoscenze sull'argomento ma che, in alcuni casi, sono rimasti all'interno della storia delle relazioni internazionali, senza proporre una riflessione sulla cultura politica dei soggetti presi in esame, e senza indagare i nessi fra ideologie di appartenenza e scelte politiche. La questione ebraica ha così finito per identificarsi con il conflitto fra Israele e i paesi arabi, e l'essere comunista o socialista è stato ridotto all'adesione a un partito e non analizzato come espressione di una visione del mondo che ha determinato concretamente le scelte di milioni di persone. In effetti, in entrambi i settori non c'è uniformità né nella scelta degli argomenti, né sui periodi studiati: mentre abbiamo diverso materiale sulla sinistra di fine secolo, anche perché fu allora che i socialisti iniziarono a tematizzare la questione ebraica, i rapporti fra la sinistra e gli ebrei durante il fascismo hanno suscitato un interesse decisamente scarso. Così, a parte qualche eccezione, non conosciamo la riflessione dei marxisti italiani degli anni Sessanta e Settanta sulla questione ebraica.

Come hanno evidenziato Edmund Silberner, George Mosse e Enzo Traverso, è esistito un problema nella definizione degli ebrei da parte della sinistra, da Marx a giorni recenti. In nome dell'internazionalismo proletario e della tradizione del movimento operaio, diversi intellettuali e politici di sinistra negarono specificità al problema ebraico, immaginando di risolverlo all'interno di un più generale progetto di cambiamento della società, che avrebbe riguardato gli oppressi di tutto il mondo, indipendentemente dalla loro identità culturale, religiosa, linguistica e nazionale. Il filosofo tedesco Karl Löwith, nel 1949, notava che, pur nelle sue molteplici espressioni, e nelle diverse fasi della sua evoluzione, il socialismo è stato una grande filosofia della storia che ha accomunato quanti hanno creduto e cercato di dare concretezza al suo progetto di trasformazione della realtà. Nei centocinquanta anni della loro esistenza, i socialisti hanno immaginato di liberare il genere umano dalla sofferenza e dalla schiavitù; e, come eredi della cultura ebraico-cristiana, e dell'illuminismo, hanno prodotto una visione del mondo universalistica e finalistica per cui l'emancipazione di tutti gli esseri umani è il significato e lo scopo ultimo della storia e dell'azione politica ${ }^{65}$.

Partendo da questa riflessione di Löwith, è possibile chiedersi chi siano gli ebrei per i socialisti: sono oppressi, e quindi insieme a tutti gli sfruttati partecipano alla lotta per l'avvento di un nuovo mondo, oppure ostacolano

${ }^{65} \mathrm{~K}$. Löwith, Significato e fine della storia. I presupposti teologici della filosofia della storia, Net, Milano, 2004, pp. 53-72. 
la realizzazione del socialismo perché rivendicano la loro identità, a partire da una religione? Non si tratta soltanto di capire in che modo gli ebrei si relazionassero al movimento operaio, ma di analizzare, come fece Mosse nel 1971, i comportamenti degli esponenti della sinistra rispetto alla comunità ebraica, in un dato momento $\mathrm{e}$ in un luogo determinato. In Italia questo approccio continua ad essere poco più che ignorato. Eppure gli autori citati ci lasciano un'indicazione molto chiara: ricostruire le relazioni politiche e culturali che la famiglia della sinistra, nelle sue diverse espressioni, ha avuto con gli ebrei è necessario per chi voglia conoscere la storia degli uni e degli altri e per occuparsi di uno dei grandi temi del Novecento, quello del rapporto fra il più importante progetto politico rivoluzionario dell'età contemporanea e la minoranza più antica del mondo. 